\title{
Esophageal metastasis secondary to extranodal nasal-type natural killer/T-cell lymphoma: A case report
}

\author{
ZHENG-YING MO ${ }^{1}$, PING WANG ${ }^{2}$, HONG-WEI YANG ${ }^{3}$, WEN-BIN LI $^{3}$ and QING-LE LIANG ${ }^{3}$ \\ ${ }^{1}$ Department of Oncology, Tai-He Hospital; ${ }^{2}$ Department of Radiology, Ren-Ming Hospital; ${ }^{3}$ Department of \\ Clinical Laboratory, Tai-He Hospital, Hubei University of Medicine, Shiyan, Hubei 442000, P.R. China
}

Received June 3, 2015; Accepted August 4, 2015

DOI: $10.3892 / \mathrm{mco} .2016 .861$

\begin{abstract}
We herein report a case of recurrent nasal natural killer (NK)/T-cell lymphoma in a 21-year-old male patient. The patient presented with an esophageal mass, fever and difficulty in swallowing. There were no other obvious sites of recurrence apart from the esophageal lesion. Metastatic esophageal lesions are extremely rare. The histological analysis demonstrated a highly aggressive tumor with a characteristic angiodestructive growth pattern and nasal cavity necrosis. The lymphoma cells were immunopositive for leukocyte common antigen, T-cell intracytoplasmic antigen 1 and CD68, negative for CD56 and CD3, and positive for Epstein-Barr virus. A computed tomography scan revealed mild thickening of the wall of the lower esophagus. The barium swallow revealed stiffness of the esophageal wall, with limited expansion and mucosal damage. The final diagnosis was primary nasal NK/T-cell lymphoma, with metastasis to the esophagus. Clinically, it is important to distinguish nasal-type NK/T-cell lymphoma from other types of tumors, as its prognosis and treatment of secondary metastases differ significantly.
\end{abstract}

\section{Introduction}

Extranodal nasal-type natural killer (NK)/T-cell lymphoma is a rare malignancy, exhibiting a high prevalence among Asians, including Chinese, Japanese and North Korean, as well as native South American populations, but is uncommon among European and North American individuals (1). NK/T-cell lymphoma is characterised by an angiodestructive growth pattern and usually diagnosed in adult, middle-aged individuals, with a male:female ratio of 2-4:1. This disease has been found to be significantly associated with Epstein-Barr virus (EBV) infection. The most common site of involvement is the nasal cavity and/or upper aerodigestive tract, and other

Correspondence to: Dr Qing-Le Liang, Department of Clinical Laboratory, Tai-He Hospital, Hubei University of Medicine, 32 S Renmin Road, Shiyan, Hubei 442000, P.R. China

E-mail: liangqingle@gmail.com

Key words: natural killer/T-cell lymphoma, extranodal type, distant metastasis midline facial structures. The most frequent sites of recurrence are the soft tissues, skin, gastrointestinal tract and testis $(2,3)$, whereas metastatic esophageal lymphoma is extremely rare. The prognosis of EN-NK/T-NT varies, mostly depending on clinical factors, including International Prognostic Index, clinical stage, applied treatment, proliferation rate and primary tumour location. Although extranodal NK/T-NT is sensitive to radiotherapy, it is inherently resistant to chemotherapy due to the expression of P-glycoprotein, and consequently, it is associated with a poorer prognosis compared with other types of lymphoma $(4,5)$. We herein report a case of extranodal nasal-type NK/T-cell lymphoma in a patient presenting with an esophageal mass.

\section{Case report}

Clinical history. A 21-year-old man, who had been diagnosed in 2011 with nasal NK/T-cell lymphoma, was admitted to the Department of Oncology at Tai-He Hospital (Hubei, China). The patient was treated with 4 cycles of cyclophosphamide, vincristine, daunorubicin and dexamethasone (CHOP regimen), followed by local radiotherapy with 56 Gy in 26 fractions (2.15 Gy/fraction) of gross tumor volume. The patient remained in remission until April, 2014, when he was admitted with a 2-month history of discontinuous fever peaking to $39^{\circ} \mathrm{C}$ without an obvious cause, and difficulty in swallowing. The patient underwent an emergent esophagogastroduodenoscopy, which revealed an irregular mucosal elevation at a distance of $30-36 \mathrm{~cm}$ from the incisors. The mucosal surface was eroded and mildly indurated. A computed tomography scan revealed a mild thickening of the wall of the lower esophagus (Fig. 1). The barium swallow indicated slow passing of the barium through the lower esophagus (equivalent to the level of the thoracic vertebrae 7-9), with esophageal wall stiffness, mildly limited expansion and mucosal damage; the length of lesion was $\sim 8.1 \mathrm{~cm}$ (Fig. 2). The patient reported no symptoms of cough, expectoration, hemoptysis or abdominal pain.

Pathological findings. The tumor cells exhibited a notable angioinfiltrative growth pattern, with homocentric arrangement around small arteries. The lymphoma cells were densely assembled and displayed abundant cytoplasm, enlarged nuclei and several large nucleoli. Mitotic figures were infrequent. 

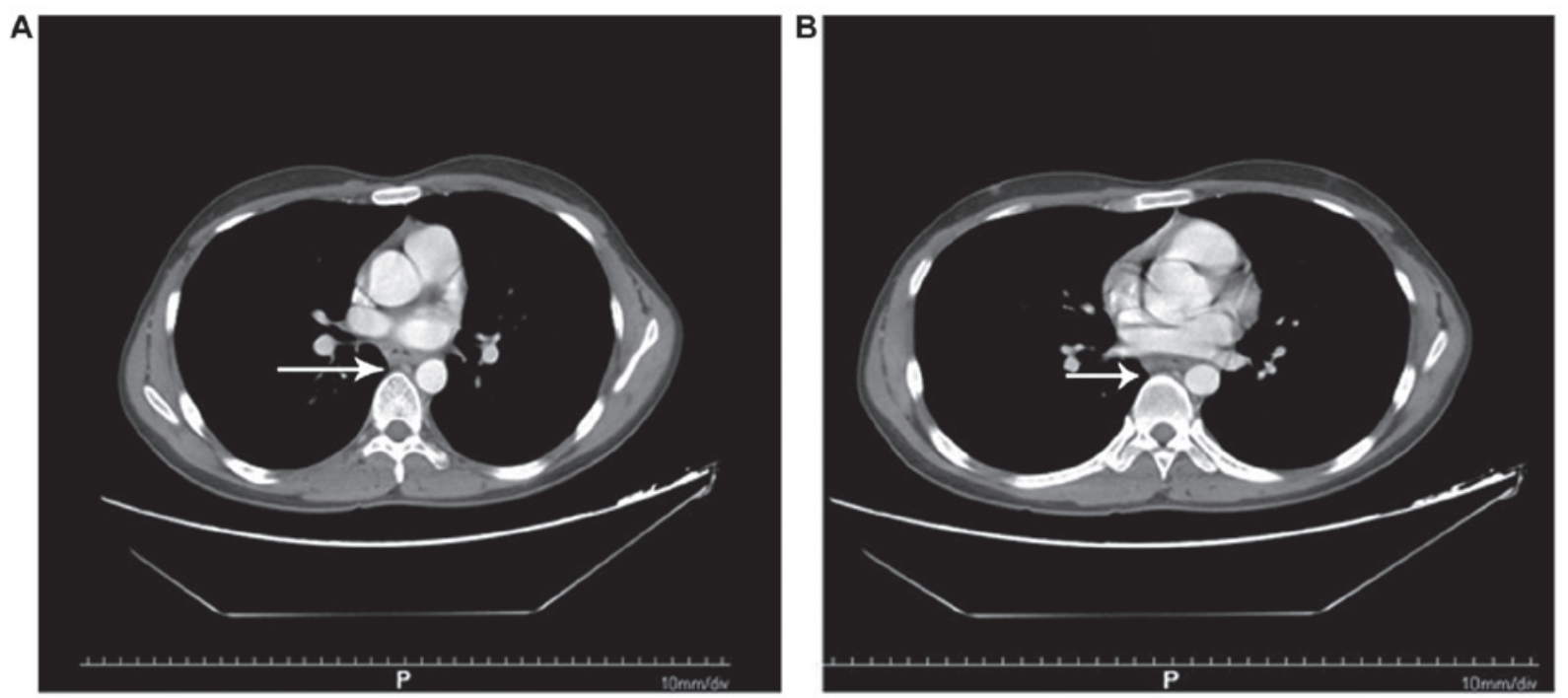

Figure 1. Computed tomography scans revealed mild thickening of the wall of the lower esophagus (arrows). (A) Before and (B) after treatment.
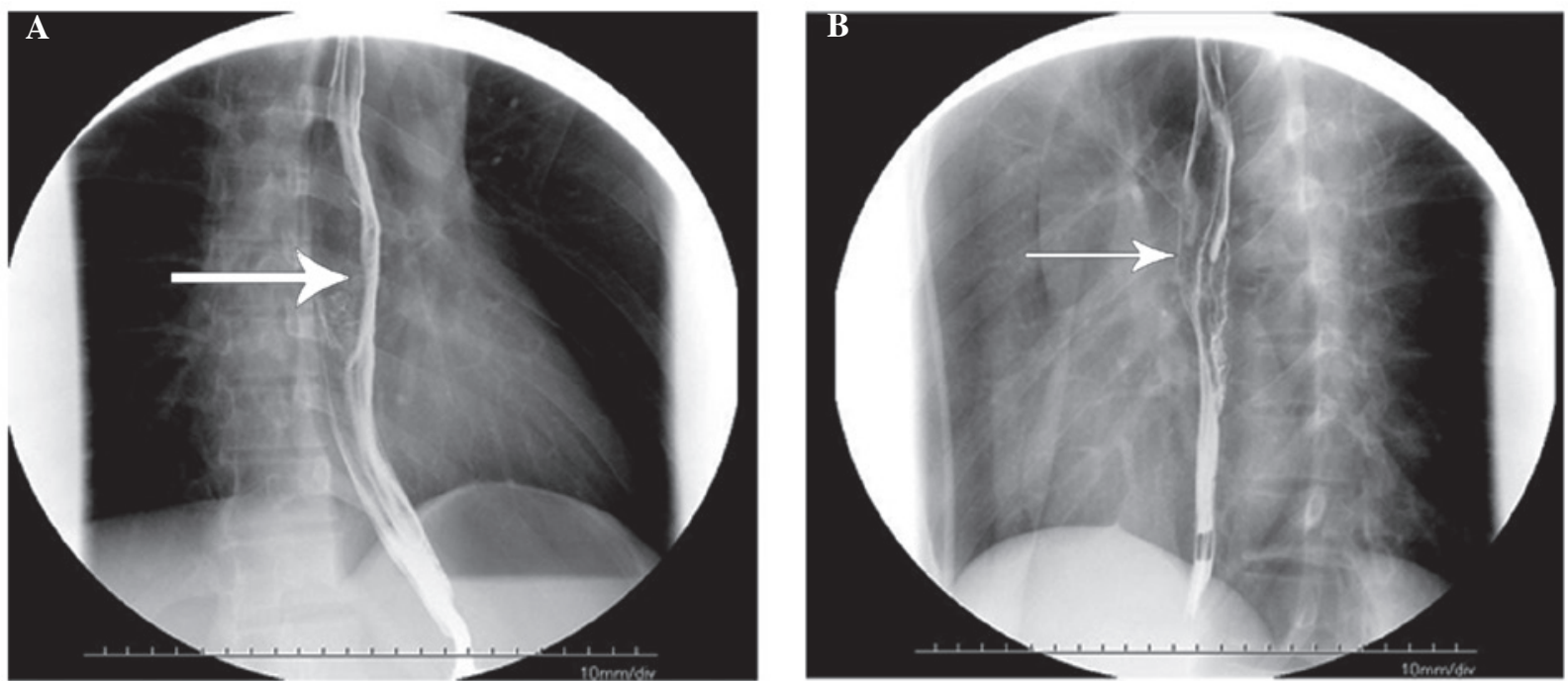

Figure 2. The barium swallow indicated slow passing of the barium through the lower esophagus (equivalent to the level of the thoracic vertebrae 7-9), with esophageal wall stiffness, mildly limited expansion and mucosal damage; the length of lesion was $\sim 8.1 \mathrm{~cm}$ (arrow). (A) Before and (B) after treatment.

Immunohistochemically, the neoplastic cells were leukocyte common antigen (LCA) ${ }^{+}$, B-cell lymphoma 6 protein $^{+}$, T-cell intracytoplasmic antigen 1 (TIA-1) ${ }^{+}, \mathrm{CD}^{+} 8^{+}, \mathrm{Ki}-67^{+}(30 \%)$, $\mathrm{EBV}^{+}, \mathrm{P}^{-} 3^{-} \mathrm{CD}^{\circ} 6^{-}, \mathrm{CD}^{-}, \mathrm{CD}^{-} 0^{-}$and cytokeratin 5/6 ${ }^{-}$. Based on the endoscopic, computed tomography and immunophenotypical findings, the diagnosis of esophageal metastasis from extranodal nasal-type NK/T-cell lymphoma was established.

The patient declined further treatment following diagnosis, due to financial difficulties, and there have been no follow-up visits or other communication following his discharge from the hospital.

\section{Discussion}

NK cells are cytotoxic lymphocytes critical to the innate immune system, which mediate lysis of tumor cells and other infected cells (6). NK cell-derived neoplasms are classified into two types, namely aggressive NK-cell leukemia and extranodal nasal-type NK/T-cell lymphoma, according to the World Health Organization (WHO) criteria $(1,7)$. Nasal NK/T-cell lymphoma is consistently associated with EBV infection (8).

According to the WHO criteria, the diagnosis of nasal-type NK/T-cell lymphoma requires EBV positivity and the presence of cytotoxic granules (2). EBV-positive NK/T-cell lymphoma exhibiting typical clinical and morphological characteristics may be classified even if they deviate from the classical immunophenotype, e.g., CD8 positivity or CD56 negativity (9). The present case exhibited the immunophenotype of tumor cells typical of nasal-type NK/T-cell lymphoma, i.e., $\mathrm{LCA}^{+}$, TIA-1 ${ }^{+}, \mathrm{CD}^{-} 8^{+}, \mathrm{CD}^{-} 6^{-}$and $\mathrm{CD} 3^{-}$. As a hallmark of nasal-type NK/T-cell lymphoma, TIA-1 and EBV-encoded RNA were the most sensitive markers of the disease. Nasal NK/T-cell lymphoma is usually associated with EBV infection, as in the present case. A high level of 
circulating plasma EBV has been correlated with high tumor load and poorer response to treatment (10). In this case, EBV may have also been an etiological factor in the development of the nasal lymphoma.

NK/T-cell lymphoma often occurs in the nose or the upper aerodigestive tract and is associated with a worse prognosis. Other sites, including the skin, spleen, salivary glands, gastrointestinal tract, lungs and testes, may also be affected (11). It has been reported that muscle tissue, the adrenal glands and the female genital tract are unusual sites of involvement (9). Bone marrow involement occurs in $<10 \%$ of patients and distant metastasis is rare. The present case was associated with a highly aggressive clinical course, with distant metastasis to the esophagus. Extranodal NK/T-cell lymphoma metastasizing to the esophagus is extremely rare and the available data on optimal treatment strategies are currently limited. Distant dissemination of nasal NK/T-cell lymphoma occurs early in the clinical course of the disease. It is crucial to distinguish nasal-type NK/T-cell lymphoma from others types, as the prognosis and treatment of secondary metastases differ significantly.

In conclusion, the present case demonstrated that primary NK/T-cell lymphoma with a poor prognosis may metastasize to the esophagus. This may represent a diagnostic pitfall that the doctor should be aware of and further consider the spectrum of differential diagnosis of esophageal tumors. Due to the clinical aggressiveness and poor prognosis of this malignancy, more effective therapeutic regiments are required for its management.

Informed consent was obtained from the family of the patient for publication of this case report and any accompanying images.

\section{Acknowledgements}

We acknowledge funds provided for outstanding young scientific and technological innovation team projects by Hubei University of Medicine (2014 CXG02).

\section{References}

1. Chan JKC, Quintanilla-Martinez L, Ferry JA and Peh SC: Extranodal NK/T-cell lymphoma, nasal type. In: World Health Organization Classification of Tumours of Haematopoietic and Lymphoid Tissues. Swerdlow SH, Campo E, Harris NK, et al (eds). IARC Press, Lyon, pp285-288, 2008.

2. Au WY, Weisenburger DD, Intragumtornchai T, Nakamura $S$ Kim WS, Sng I, Vose J, Armitage JO and Liang R; International Peripheral T-Cell Lymphoma Project: Clinical differences between nasal and extranasal natural killer/T-cell lymphoma: A study of 136 cases from the International Peripheral T-Cell Lymphoma Project. Blood 113: 3931-3937, 2009.

3. Barrionuevo C, Zaharia M, Martinez MT, Taxa L, Misad O, Moscol A, Sarria G, Guerrero I, Casanova L, Flores C, et al: Extranodal NK/T-cell lymphoma, nasal type: Study of clinicopathologic and prognosis factors in a series of 78 cases from Peru. Appl Immunohistochem Mol Morphol 15: 38-44, 2007.

4. Suzuki R, Takeuchi K, Ohshima K and Nakamura S: Extranodal NK/T-cell lymphoma: diagnosis and treatment cues. Hematol Oncol 26: 66-72, 2008.

5. Liang L, Nong L, Zhang S, Zhao J, Ti H, Dong Y, et al: The downregulation of PRDM1/Blimp-1 is associated with aberrant expression of miR-223 in extranodal NK/T-cell lymphoma, nasal type. J Exp Clin Cancer Res 33: 7, 2014.

6. Spits H, Lanier LL and Phillips JH: Development of human T and natural killer cells. Blood 85: 2654-2670, 1995.

7. Chan JKC, Jaffe ES, Ralfkiaer E and Ko YH: Aggressive NK-cell leukaemia. In: WHO Classification of Tumours of Haematopoietic and Lymphoid Tissues. International Agency for Research on Cancer, Lyon, France, pp276-277, 2008.

8. Chan JKC, Jaffe ES and Ralfkiaer E: Extranodal NK/T-cell lymphoma, nasal type. In: World Health Organization Classification of Tumors. Pathology \& genetics: Tumors of hematopoietic and lymphoid tissues. Jaffe ES, Harris NL, Stein H, et al (eds). IARC Press, Lyon, France, pp204-207, 2001.

9. Hasserjian RP and Harris NL: NK-cell lymphomas and leukemias: A spectrum of tumors with variable manifestations and immunophenotype. Am J Clin Pathol 127: 860-868, 2007.

10. Au WY, Pang A, Choy C, Chim CS and Kwong YL: Quantification of circulating Epstein-Barr virus (EBV) DNA in the diagnosis and monitoring of natural killer cell and EBV-positive lymphomas in immunocompetent patients. Blood 104: 243-249, 2004.

11. Cheung MMC, Chan JKC, Lau WH, Foo W, Chan PT, Ng CS and Ngan RK: Primary non-Hodgkin's lymphoma of the nose and nasopharynx: Clinical features, tumor immunophenotype, and treatment outcome in 113 patients. J Clin Oncol 16: 70-77, 1998. 